\title{
An Analytical Model for Molecular Propagation in Nanocommunication via Filaments Using Relay-Enabled Nodes
}

\author{
Kamaloddin Darchinimaragheh and Attahiru S. Alfa
}

\begin{abstract}
Molecular communication is a nanoscale communication method in which information is encoded in molecules. Molecular communication using microtubules in free space is one of the realistic scenarios proposed for this type of nanocommunication. Based on this technique, molecular communication via filaments using nano-relays is proposed in this paper to improve the performance of the system in terms of delay and bit error probability. An analytical model using jump diffusion processes is proposed for molecular propagation in this scenario. It is shown that the proposed mathematical model is capable of tracking molecular propagation in molecular communication via filaments using nano-relays. Also, the model is used to investigate effects of different parameters on delay and bit error probability.
\end{abstract}

Index Terms-Filament, jump diffusion process, molecular communication, nano-network.

\section{INTRODUCTION}

$\mathbf{R}$ ECENT advances in nanotechnology promise our ability of building functional units capable of performing simple tasks at molecular level. At this scale, we call these units nanomachines. In order to perform complex tasks, nano-machines cooperate with each other and constitute networks. Such networks are called nano-networks [1].

In order to constitute a nano-network, nano-machines should communicate. However, due to the size of nano-machines it is not feasible to use classical communication methods. Therefore, novel communication methods compatible with the properties of nano-networks are proposed. A bioinspired and promising solution is molecular communication. In this type of nanocommunication, information is encoded in molecules.

Nanocommunication via diffusion, nanocommunication via physical contact, and nanocommunication via microtubules are different types of molecular communication [2]. In nanocommunication via diffusion, a transmitter emits molecules which diffuse through the medium, reach the receiver, and bond to the

\footnotetext{
*K. Darchinimaragheh is with the Department of Electrical and Computer Engineering, University of Manitoba, Winnipeg, MB R3T 5V6, Canada.

A. S. Alfa is with Department of Electrical and Computer Engineering University of Manitoba, Winnipeg, MB R3T 5V6, Canada, and Department of Electrical, Electronic and Computer Engineering University of Pretoria, Pretoria 0002 , South Africa.
}

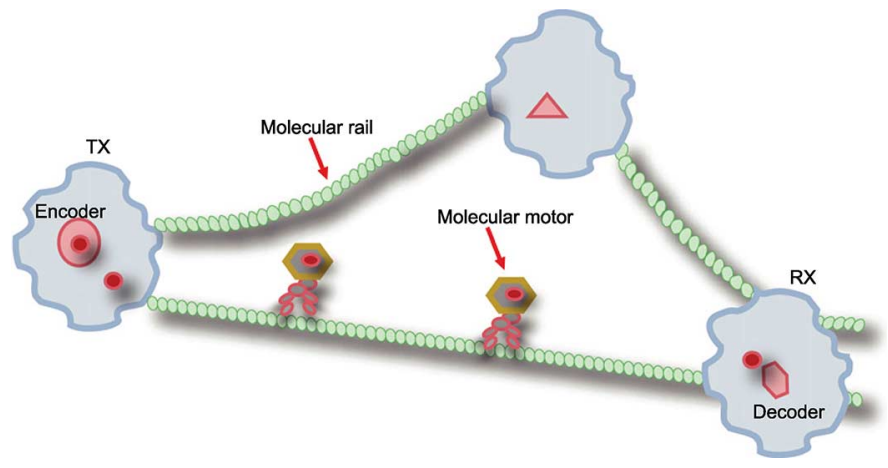

Fig. 1. A sample illustration for molecular communication via microtubules [1].

receptors on the receiver which results in receiving the message. Nanocommunication via physical contact is divided into two subcategories: nanocommunication via gap junctions, and nanocommunication via synapses. In the former case, gap junctions are used to transmit information molecules from a transmitter to the receiver, and in the latter case synapses are used. In nanocommunication via microtubules cargo transmission is carried out by binding the cargo to a special polymer, called molecular motor, which is capable of attaching to and moving along cylindrical polymers, called microtubules, while carrying the cargo. Fig. 1 illustrates an example for this type of molecular communication. A comprehensive study of molecular communications can be found in [2]-[4].

There have been many research works on nanocomunication using microtubules. Farsad et al. consider a bounded channel covered with motors on which microtubules move from a transmitter to the receiver (inverted geometry) [5]. They find the mutual information and show that the number of times a microtubule can move between a transmitter and the receiver in inter-symbol time is an important parameter determining mutual information of the system. Then, they extend their work by considering a drift for microtubules [6], and proposing a semi-anaytic model to calculate the mutual information [7]. Finally, they suggest a method to find the optimal channel dimensions for a given inter-symbol time [8].

Moore et al. propose different scenarios for molecular communication using microtubules. They consider a single receiver to which 4 microtubules are attached and find the probability of sending an information molecule to the receiver [9]. Then, they extend the scenario by considering several receivers, and also polarity for microtubules [10]. Moore et al. find the probability of receiving information molecules and information rate of the scenarios in [11], and propose approaches to maximize receiving probability and information rate of the systems in [12]. 
A comprehensive survey on nanocommunication using microtubules, and generally molecular communication, can be found at [2].

We call the most advanced scenario proposed by Moore $e t$ al. nanocommunication in free space [2]. In this scenario, a number of microtubules are attached to each nano-machine. The transmitter releases an information molecule attached to a motor. The unit diffuses through the medium and in case it reaches a microtubule, the motor will attach to the microtubule and move along it towards the receiver, carrying the information molecule [9]. Upon receiving the unit, the receiver will remove it from the channel. Guiding motors towards the receiver is the main advantage of using microtubules. Here, we note that throughout this work we use the terms diffusion process and Brownian motion interchangeably.

Reliability is of fundamental importance in implementing molecular communication. However, nanocommunication via microtubules in free space is reliable only if a transmitter and the receivers are close [10], [9], [11]. The techniques proposed to improve the reliability are mostly for nanocommunication via diffusion. Unluturk et al. suggest to use network coding and show that it improves the rate for a given delay and vice versa [25]. In another work, Aijaz et al. investigate error performance of network coding in molecular communication via diffusion and derive a closed form for error probability [26]. To improve the performance of nanocommunication via microtubules, borrowing the idea from wireless communication networks, we propose another approach and deploy nano-relays. Nano-relays are nano-machines which are capable of receiving motors and directing them towards receivers.

Microtubules are used in most of the research works which has been done. However, actin filaments are an alternative. In [27], a comprehensive study of using actin filaments and Myosins for cargo transportation is presented. Also, the term active molecular communication is used in [28] to refer to both nanocommunication using microtubules and actin filaments, and continuous and discrete models for motors moving along a filament is presented. In this paper, we focus on message (cargo) propagation using actin filaments, since myosins move faster on filaments than kinesins and dyneins on microtubules. With these proposals, the considered scenario will be as follows. A number of filaments are attached to each and every nano-relay, and nano-relays are distributed in the channel randomly. If a motor attaches to a filament, it will be directed towards the receiver. This type of movement is different from random movement resulting from diffusion. The oriented movement of messages reduces the delay and improves the reliability of the communication technique. We will call this technique molecular communication via filaments using nano-relays.

The contributions of this paper can be summarized as follows:

- Deploying nano-relays to improve reliability of the nanonetwork.

- Proposing an analytical model for molecular propagation in molecular communication via filaments using nano-relays.

The rest of the paper is organized as follows. In Section II, we discuss molecular communication via filaments using nano-relays. Then in Section III, we discuss the approximation method for jump diffusion processes, and in Section IV, the necessary parameters are calculated to use the approximation method to model molecular propagation in molecular communication via filaments using nano-relays. Section $\mathrm{V}$ presents the results, and finally, Section VI concludes the paper.

\section{Molecular Communication VIA Filaments Using NANO-RELAYS}

Consider a channel with one transmitter which releases an information molecule already attached to a molecular motor (in the rest of this paper, we simply use motor to refer to information molecule-molecular motor pair). To elaborate on the performance improvements of the proposed technique, we consider a bounded two-dimensional channel where the transmitter is at the middle of it (equal distance to the boundaries). Also, the two boundaries on the two sides of the channel as receivers. If a motor hits the other boundaries it will be reflected. Also, all the nodes have relaying capability. Assume an average of $N$ nano-relays are distributed in the channel based on a Poisson point process, and $M$ filaments are attached to each of them. The orientations of all the filaments attached to a nano-relay are uniformly distributed between 0 and $2 \pi$ (with respect to the $x$ axis) except for one filament which is oriented towards the closest receiver (if a nano-relay has equal distances to receivers, we pick one receiver at random as the closest receiver). If a nano-relay receives a motor, instead of removing the motor, it will put the motor on this filament. This will guide the motor towards the closest receiver.

We have assumed nano-relays are capable of distinguishing a filament, and also, putting a motor on a specific filament. An example for the former assumption (distinguishing a filament) is the transmigration across endothelial cells (cells that line the interior surface of blood vessels) [14], [15]. The nucleoid of these cells receives cargoes from filaments attached to one side of it and picks one of the filaments leading to a blood vessel to put the motor on, and consequently, sends the cargo into blood stream. In addition to this example, a practical technique is using concentration gradient. Assuming that the receivers emit a specific type of molecules, the concentration of the molecule is higher on the receiver side and it can be sensed by nano-relays. We propose a high level model in this paper and assume this process is performed in an ideal manner. The latter assumption can be achieved if nano-relays release motors and the cargo at a vicinity of the intended filaments. The higher the concentration of motors at the vicinity of the filament is, the higher the probability of the cargo attaching to a motor and the motor attaching to the filaments. In this paper, for tractability reasons, we assume that the concentration of motors is high enough and probability of attaching to the filament is 1 .

There are different types of molecular motors. Attaches to a filament, a molecular motor may move towards the $+v e$ end or the -ve end of the filament, depending on the type of the motor. For example, Myosin 6 move towards -ve end of a filament and most other myosins move toward the +ve end [17]. It is important to note that the + and - signs used here do not refer to electrical charges. They simply refer to motors' directions of movement, which depends on the structure of filaments and motors. This notation is borrowed from [10]. Assume each nano-relay is located either on the +ve end ( $\mathrm{R}+$ topology) or on the -ve end (R-topology) of all of the filaments attached to it. If a nano-relay in an $\mathrm{R}+$ topology receives a motor, since the nano-relay is on the +ve side of all the filaments attached to it, the motor has moved towards the +ve end of the filament. 
Having received a motor, the nano-relay should bond the received information molecule to a motor and put it on the filament directed towards the receiver. However, this time the motor is supposed to move towards the -ve end of the filament (away from the nano-relay). Since the received motor moves towards the $+v e$ end of a filament, the nano-relay should use a different type of motor which moves towards the -ve end of a filament. We assume each nano-relay stores as many motors as needed, and it is capable of breaking the bond between an information molecule and a motor, and making a new one between the information molecule and a new motor. A comprehensive discussion on how these processes could be done and what are the necessary conditions on the information molecules and the motors can be found at [18]. Half of the nano-relays have R+ and the other half have R-configurations in the scenario .

Consider a motor which moves towards -ve end of a filament. Being released from the transmitter, the motor diffuses through the medium and in case it reaches a filament, it will attach to the filament. If the motor attaches to a filament belonging to a nanorelay in an R- topology, the motor will start moving towards the nano-relay. However, if the motor attaches to a filament in an $\mathrm{R}+$ topology, it will move towards the -ve end of the filament and away from the nano-relay. These two scenarios are treated separately in the model.

Without loss of generality, we assume filaments are straight. If the filaments are not straight, the jump distribution would change. However, the same approach can be applied to find the model for molecular propagation. Also, it is assumed that filaments are shorter than the average distance between neighbor nano-relays and longer than half the distance. If filaments are shorter diffusion will be the dominant process for propagation and therefore, using the proposed technique is not advantageous. Fig. 3 shows a comparison for the scenario explained in Section V between filaments' length of $10 \mu \mathrm{m}$ and $5 \mu \mathrm{m}$ and free diffusion (in the considered scenario, average distance between nano-relays is $11.2 \mu \mathrm{m}$ ). It can be seen in the figure that performance of the former scenario is close to performance of free diffusion in terms of delay. On the other hand, if filaments are longer than the range, there will be several crossovers. Since the model proposed in this paper does not consider crossovers, the results for such a scenario will not be accurate. Additionally, we assume all the filaments have the same length. Future works can relax these assumptions and make the model more general. Fig. 2 shows the scenario for eight nano-relays, i.e., $N=8$, in the channel with four filaments, i.e., $M=4$.

As long as a released motor is not attached to a filament, it freely diffuses through the medium. If it reaches a filament, depending on its type it will start walking towards the nano-relay or the other end of the filament. Either way, the motor will reach the end of a filament after some time and diffuse again. So, if we look at the whole process of a motor's movement in the medium, it is a diffusion process with discontinuities. In other words, the attaching and detaching processes will cause discontinuities in the diffusion process and jumps in the position of the motor. This leads to the idea of using jump diffusion processes.

We note that not all released motors reach a filament. However, considering that the length of filaments are not too short (compared to the average distance between nano-relays), it happens with a high probability. Results in Section V illustrate that. Also, the proposed model is probabilistic. Therefore, it does not

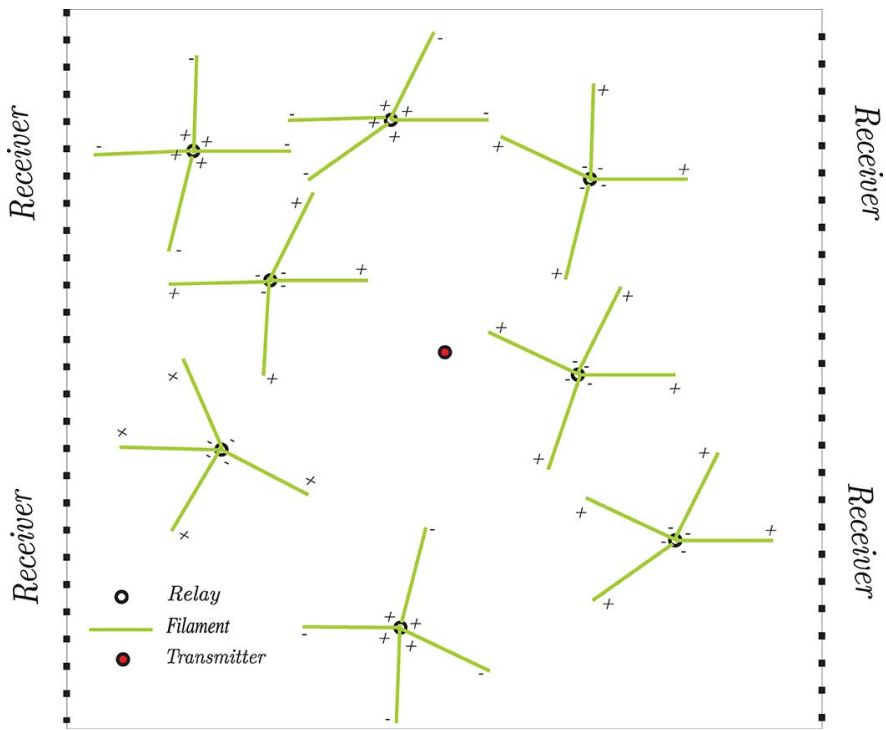

Fig. 2. A sample scenario. The transmitter is in the middle of the channel, the two right and left sides of the channel are considered as receivers, and the nanorelays are distributed in the channel. There are four filaments attached to each nano-relay.

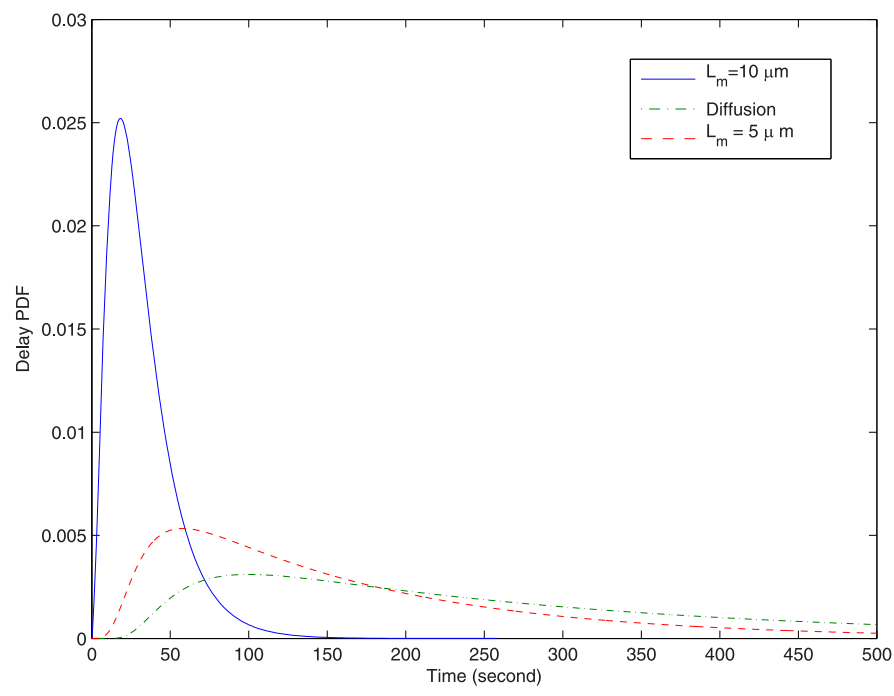

Fig. 3. Comparison between three scenarios: filaments are long, filaments are short, and free diffusion.

assume that all information molecules attach to filaments with probability 1 .

\section{DTMC APPROXIMATION FOR JUMP DiffUSION ProcesSes}

In this section, we present an approximation method for jump diffusion processes based on discrete time Markov chains (DTMC). We start with a brief introduction of Brownian motion. Then, the approximation method is discussed for the simple case of one-dimensional Brownian motion. Next, the model is extended to a two-dimensional process, and finally, the method is modified to approximate a jump diffusion process.

\section{A. Brownian Motion Approximation}

In this section, we first introduce Brownian motion briefly. Then, we study the DTMC approximation for Brownian motion without drift. The detailed explanation of the model can be found in [20]. Brownian motion is the mathematical model 
for free movement of particles. The one-dimensional probability distribution of the displacement of a particle diffusing in a medium is time dependent, and is formulated in the form of [13]:

$$
f(\Delta x, \Delta t)=\frac{1}{\sqrt{2 \pi D \Delta t}} \exp \left(\frac{-\Delta x^{2}}{2 D \Delta t}\right),
$$

where $\Delta x$ stands for the relocation, and $\Delta t$ for the time period. Also, $D$ is the diffusion coefficient of the medium.

Assume $D$ is 1. At the end of this section, we will relax this assumption. Consider a one-dimensional space which is bounded by $X_{1}$ and $X_{2}\left(X_{1}<X_{2}\right)$. Since the goal is to use a Markov chain, we convert the continuous space into a discrete one by dividing it into $m$ intervals ( $m$ is a large number, and the larger the $m$ the more accurate the model. For a given error, a sample scenario can be considered to find minimum $m$ which satisfies the error. Minimum $m$ minimizes the size of the problem [20]) with equal lengths of $\Delta x=h / m$, where $h=X_{2}-X_{1}$. Additionally, we discretize time into intervals of $\Delta t=m^{2} / h^{2}$. Based on (1), it is shown in [20] that the probability distribution of the displacement of a diffusing particle in the discrete space in $\Delta t, X$, is equal to:

$$
P(X=k \Delta x)= \begin{cases}\frac{C^{-1}}{\sqrt{2 \pi}} \exp \left(\frac{-k^{2}}{2}\right) & k \neq 0 \\ \frac{C^{-1}}{\sqrt{2 \pi}} \sum_{\substack{l=-\infty \\ l \neq 0}}^{l=+\infty}\left(l^{2}-1\right) \exp \left(-\frac{l^{2}}{2}\right) & k=0,\end{cases}
$$

where $C$ is a normalization factor and equal to:

$$
C=\frac{1}{\sqrt{2 \pi}} \sum_{\substack{l=-\infty \\ l \neq 0}}^{l=+\infty}\left(l^{2}\right) \exp \left(-\frac{l^{2}}{2}\right) .
$$

Now, consider a Markov chain whose states are the spatial intervals introduced earlier $(\Delta x)$ and the transition probabilities are defined based on (2). Therefore, the entries of the corresponding transition matrix of the Markov chain, $T$, are:

$$
T_{i j}=P\left(X_{t+1}=j \Delta x \mid X_{t}=i \Delta x\right), i, j=1,2, \ldots, m,
$$

where $X_{t}$ is the state of the Markov chain at time $t$. This probability can be calculated by putting $k=j-i$ in (2).

It is shown in [20] that if:

$$
W(t)=\text { state of the Markov chain at } t,
$$

then:

$$
W(t) \rightarrow B(t), \text { as } m \rightarrow \infty,
$$

where $B(t)$ is standard Brownian motion. In other words, the Markov chain approximates the position of a diffusing particle at $t=n \Delta t, n=0,1, \ldots$ It is worth mentioning that since the probability distribution in (1) is not a function of $x$ (it is a function of $\Delta x$ ), the transition probabilities presented for the Markov chain are not a function of $X_{t}$ or $X_{t+1}$. They are only a function of $X_{t+1}-X_{t}$.

In order to be comprehensive, we extend the model to a twodimensional space. We build a Markov chain with state space $(X, Y)$ where $X$ defines the $x$ component and $Y$ defines the $y$ component of the position of a particle. Since $x$ and $y$ components of a diffusion process are independent and the transition probabilities for both components follow (2), the transition matrix of the Markov chain will be:

$$
\hat{T}=T \otimes T,
$$

where $T$ is defined in (4).

Finally, what if $D \neq 1$ ? It is stated in [20] that one can still assume $D=1$, and modify the boundary of the channel instead. If $R$ stands for the boundary of the actual channel, the boundary of the modified channel will be $(1 / \sqrt{D}) R$. We will use this technique in this paper.

\section{B. Jump Diffusion Process Approximation}

In this section, we first define jump diffusion process briefly. Then, we extend the DTMC approximation method discussed in Section III-A for jump diffusion processes [21].

A jump diffusion process is defined as a Brownian motion with discontinuities (or jumps). Mathematically, it is of the form:

$$
X_{t}=\sigma B(t)+\mu t+\sum_{i=1}^{N(t)} J_{i},
$$

where $B(t)$ is Brownian motion with zero drift and variance $1, \sigma$ is the square root of variance of $B(t), \mu$ is the drift of the process, $N(t)$ is a Poisson process with rate $\lambda$, and jump sizes $\left\{J_{i}, i=1,2, \ldots\right\}$ are independent identically distributed random variables. Without loss of generality, we assume $\sigma=1$, and considering the scenario, $\mu=0$. Also, we assume $B(t)$, $N(t)$, and random variables $\left\{J_{i}, i=1,2, \ldots\right\}$ are independent. It should be noted that since $B(t)$ is a Markov process, $N(t)$ has Poisson distribution, and $J_{i}$ s are independent, $X(t)$ is also a Markov process.

Unfortunately, jump diffusion processes have not been investigated thoroughly yet. Specifically, if jump sizes are not independent double exponential random variables (one exponential random variable if the jump is in the positive direction, and another exponential random variable if the jump is in the negative direction), formulating the process will be complex [22]. Some research works have considered jump distributions other than double exponential, such as hyper-exponential [23], but considering an arbitrary jump size makes it very complex. Since the jump sizes in the considered scenario in this paper have arbitrary distribution, we introduce an approximation method for the diffusion jump process and use the method to calculate the position distribution and boundary crossing probability of the process, which is mapped into the delay of information molecules.

In order to use the approximation method introduced in Section III-A for jump diffusion processes, we discretize time. Consequently, inter-jump intervals have geometric distribution (with parameter $\lambda \Delta t$ ) and jumps happen only at the end of a time unit. Also, assume:

$$
P(J=k \Delta x)=J(k) .
$$

Considering spatial intervals as the states of a Markov chain, the entries of the corresponding transition matrix, $S$, are as follows:

$$
S_{i j}=(1-\lambda \Delta t) T_{i j}+\lambda \Delta t J(j-i),
$$

where $T$ is the transition matrix introduced in Section III-A.

In this paper, we assumed the two boundaries other than the receivers are reflective. Therefor, as long as receiving a motor 
is of concern, $y$ component of the position of the motor is irrelevant and we can consider a one-dimensional model. However, in order to be comprehensive, we extend the model to a two-dimensional space. We assume the $x$ and $y$ components of a jump diffusion process are independent. It should be noted that we are proposing a stochastic model. Therefore, it is not necessary to follow the exact movement of a particle. However, the model should statistically match the movement. So, it is valid to assume $x$ and $y$ components of jumps are independent given that the distributions match the distributions in the scenario. To be more specific, jumps in the $x$ and $y$ components might not happen at the same time slot, but looking at the whole process, the jumps on the $x$ and $y$ components match stochastically. Therefore, the one-dimensional approximation can be extended to approximate a two-dimensional jump diffusion process as explained in Section III-A. The transition matrix of the resulting Markov chain, $\hat{S}$, will be:

$$
\hat{S}=S \otimes S,
$$

where $S$ is given in (10).

\section{Propagation Model}

In this section, we present the propagation model for molecular communication via filaments using nano-relays. We derive the necessary parameters to use the one-dimensional approximation method discussed in Section III-B.

Inter-jump intervals and jump sizes are the two random variables that should be known to define a jump diffusion process. In this regard, we first discuss that it is reasonable to assume that the distribution of the inter-jump times is exponential, and then, find the parameter of the distribution. Next, we calculate the jump size probability distribution.

A motor which is not attached to a filament diffuses through the medium. Considering the independent increments property of diffusion, and also the assumption that nano-relays are distributed based on a Poisson point process, the remaining time to the next attachment is independent of the time passed since the last attachment of a diffusing motor. Therefore, the time interval between two consecutive attachments to filaments has an exponential distribution.

In order to find the rate of the inter-jump process, we use a known formula. It is known that the average time for a diffusing particle to pass a circle of radius $\Delta r$ in a two-dimensional space for the first time is [24]:

$$
t_{\text {avg }}=\frac{1}{2 D}(\Delta r)^{2}
$$

where $D$ is the diffusion coefficient of the medium. Therefore, if $d_{a v g}$ is the average distance a motor diffuses between two consecutive attachments to filaments, the average inter-attach interval, or equivalently inter-jump time for a motor is:

$$
t_{\text {avg }}=\frac{1}{2 D} d_{\text {avg }}^{2}
$$

and consequently, the rate of the inter-jump process will be:

$$
\lambda=\frac{1}{t_{\text {avg }}}=\frac{2 D}{{d_{\text {avg }}}^{2}} .
$$

We need to find $d_{\text {avg }}$. Since $d_{\text {avg }}$ is the average distance, we consider a symmetric channel. We divide the channel into $N$ equal squares ( $N$ is the number of nano-relays) and consider one nano-relay at the middle of each square (a symmetric

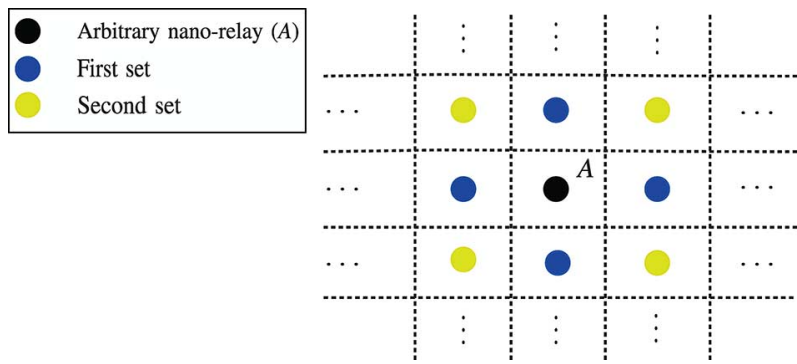

Fig. 4. Illustrating the first and second sets of neighbors for an arbitrary nanorelay named $A$.

channel in terms of the position of nano-relays). For an arbitrary nano-relay, named $A$, the four nano-relays located on its right, left, up, and down consist the first set of neighboring nano-relays. The nano-relays between each two members of the first set consist the second set, and so on. Fig. 4 illustrates a sample topology. We assume that a motor detached from $A$ will attach to a filament belonging to neighboring nano-relays of $A$. We find the number of sets of neighbors that should be considered in the model and also the probabilities of binding to each set of neighbors. As we will show, the average distance between two nano-relays that a motor attaches to their filaments consecutively is close to the average distance between $A$ and the set of its first, second, and third closest neighboring nano-relays. Therefore, we assume that a motor detached from nano-relay $A$ will attach to a filament belonging to the set of first, second, or third closest neighboring nano-relays of $A$. To derive the binding probabilities, we present simulation results for the distribution of the distance between two nano-relays that a motor attaches to their filaments consecutively. We consider a two-dimensional channel $(100 \times 100 \mu \mathrm{m})$. The size of the channel is chosen in a way to ensure that a released motor attaches to at least one filament before reaching a receiver and also diffuses after detaching from the filament. Therefore, the channel dimensions are much longer than both the average distance a motor moves between two consecutive attachments, which is $14.10 \mu \mathrm{m}$, (to ensure motors attach to at least one filament), and the length of filaments (to ensure motors are not received immediately after detaching from filaments). The rate of the Poisson point process for nano-relays distribution is $8 \times 10^{9}$. The simulation results are presented for two length of filaments: $5 \mu \mathrm{m}$ and $10 \mu \mathrm{m}$ to ensure the generality of the results. As shown in Fig. 5, the distribution of the distance between two nano-relays that a motor attaches to their filaments consecutively is close to uniform distribution. Therefore, we assume the probability of binding to the filaments of the three sets of neighboring nano-relays is the same (equal to $1 / 3$ ).

Considering the above mentioned setting, $d_{a v g, 1}, d_{a v g, 2}$, and $d_{a v g, 3}$ are the average distances of nano-relay $A$ to another nanorelay belonging to the set of $A$ 's first, second, and third closest neighbors, respectively. The average distance between $A$ and another nano-relay belonging to the set of $A$ 's closest neighbors is:

$$
d_{\text {avg }, 1}=\sqrt{\frac{X Y}{N}},
$$

where $X$ and $Y$ are the width and length of the channel. Similarly, the average distance to the set of second and third closest neighbors are:

$$
d_{a v g, 2}=\sqrt{2} d_{a v g, 1}, d_{a v g, 3}=2 d_{a v g, 1} .
$$




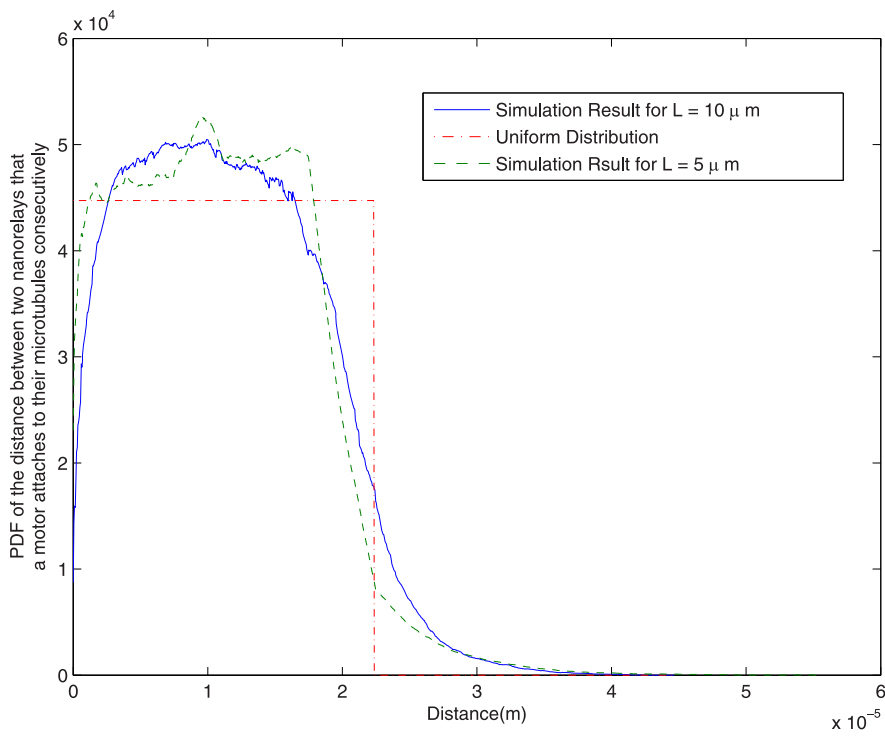

Fig. 5. Comparing the distribution of the distance between two nano-relays that a motor attaches to their filaments consecutively, and a uniform distribution in the same range.

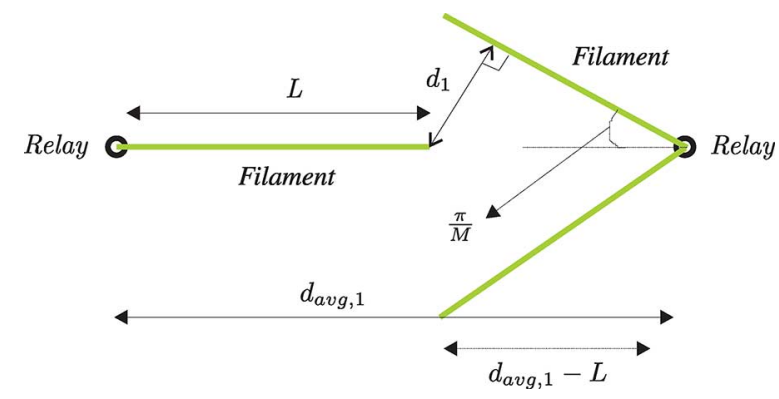

Fig. 6. The average distance a motor should diffuse to reach a nano-relay belonging to the set of the nearest neighbors.

Using this technique, for the scenario in which filaments' length is $10 \mu \mathrm{m}$ the average distance between two nano-relays that a motor attaches to their filaments consecutively is $14.10 \mu \mathrm{m}$ and $16.14 \mu \mathrm{m}$, based on simulation and analytical results, respectively. The error is $12.5 \%$, which is negligible. For the scenario in which filaments' length is $5 \mu \mathrm{m}$ the average distance based on analytic result is the same, and based on the simulation results is $13.66 \mu \mathrm{m}$. The error is $14.3 \%$, which is not as good as the other scenario, but still not huge.

If an emitted motor attaches to one of the first neighbors, based on Fig. 6 the distance it should diffuse is:

$$
d_{1}=\left(d_{a v g, 1}-L\right) \sin \left(\frac{\pi}{M}\right),
$$

where $L$ is the length of a filament, and $2 \pi / M$ is the average angle between two adjacent filaments attached to a nano-relay.

To find the average distance a motor diffuses to attach to a filament belonging to the second or third neighbors, we assume the motor attaches to the middle of the filament. Nano-relays are distributed based on a PPP, and therefore, they are uniformly distributed in the channel. Since the average distance between $A$ and another nano-relay belonging to the set of its second or third neighbors is longer than the length of filaments, the motor should diffuse throught the channel to reach the next filament. Consequently, the probability distribution of the position a motor attaches to a filament belonging to the next nano-relay is defined based on a diffusion process. Since the direction of each

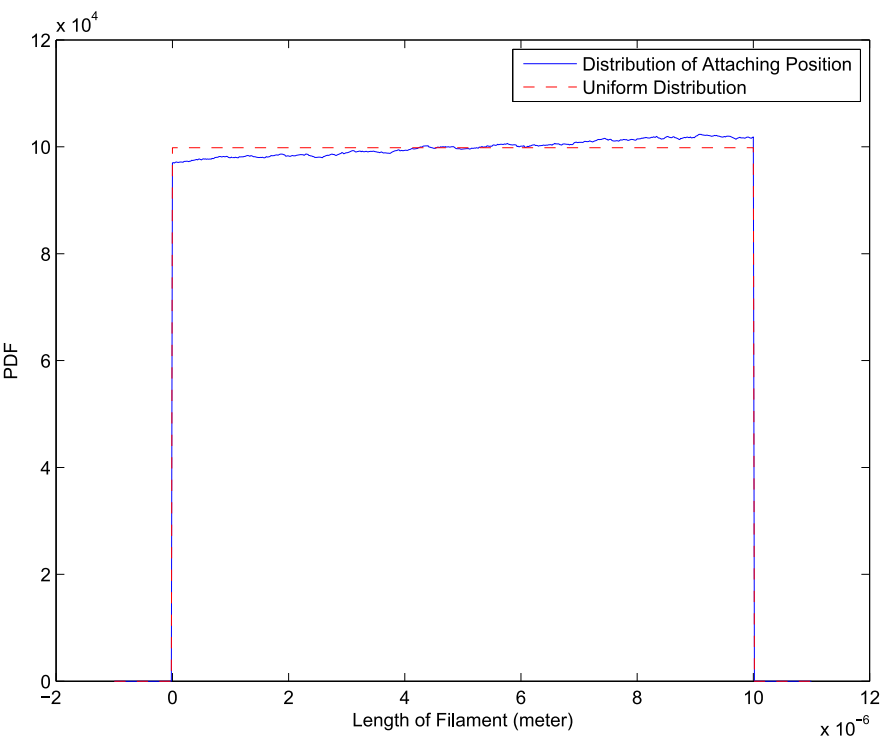

Fig. 7. consecutively the assumption that a motor attaches to the middle of the next filament. The figure compares probability distribution of the position a motor attaches to the next filament and a uniform distribution, for the same interval.

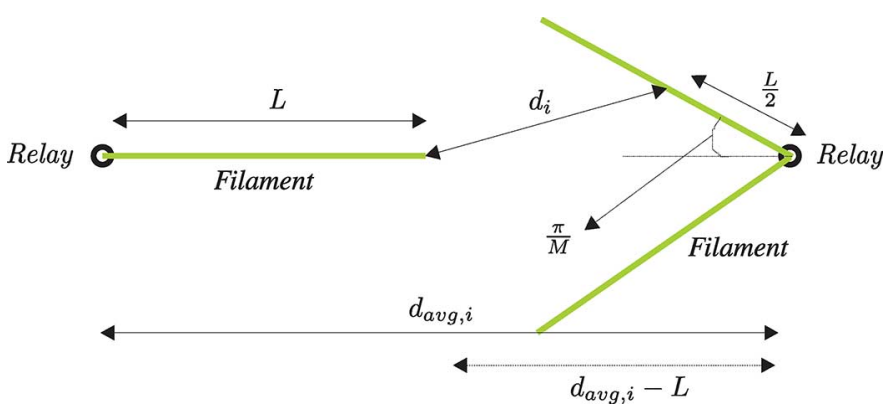

Fig. 8. The average distance a motor should diffuse to reach a nano-relay belonging to the set of the second or third nearest neighbors.

step in Brownian motion is picked based on a uniform distribution, the distribution is approximately uniform over the length of the filament. Results shown in Fig. 7 validates this assumption. Fig. 7 compares the probability distribution for the position where a motor attaches to a filament (blue) with a uniform distribution for the same interval (red). Based on this figure, the probability distribution is very close to uniform. The probability distribution deviates from the uniform distribution for up to $5.8 \%$. The results are derived for the scenario defined in Section V. Considering this assumption and Fig. 8, the average distance a motor should diffuse is:

$$
\begin{array}{r}
d_{i}=\sqrt{\left(d_{a v g, i}-\frac{L}{2}\right)^{2}+\frac{L}{2}^{2}-2\left(d_{\text {avg }, i}-\frac{L}{2}\right) \frac{L}{2} \cos \left(\frac{\pi}{M}\right)}, \\
i=2,3 .
\end{array}
$$

Therefore, the average distance a motor should diffuse to reach a filament is:

$$
D_{\text {avg } 1}=\frac{d_{1}+d_{2}+d_{3}}{3} .
$$

It is worth noting that we assumed the orientation of at least one filament of each nano-relay is fixed towards the closest receiver. Let us call such filament $M_{d}$. If polarity of a motor is in match with the filament it attaches to, the motor will detach from $M_{d}$ of the nano-relay. In this case, the probability 


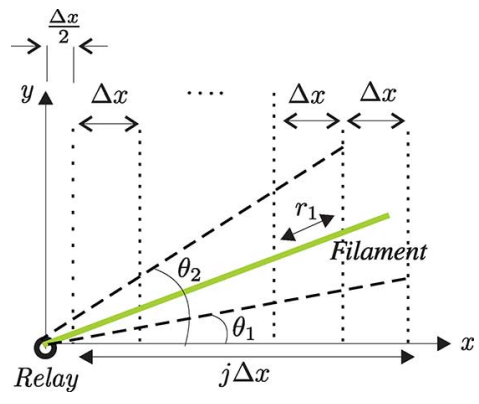

Fig. 9. Jump size of one, for an arbitrary position of a filament.

that the motor attaches to $M_{d}$ of any of the neighbor nano-relays is low since $M_{d}$ is the furthest filament and the motor should go around a nano-relay to reach it. This will modify (17) and (18) by changing $M$ to $M-1$. We call the average distance calculated for this scenario $D_{a v g 2}$. However, if the polarity of an attached motor is not in match with the filament it is attached to, (17) and (18) will remain as they are. Since the number of nano-relays in R+ and R-topology are equal and they are distributed in the channel uniformly, the average distance a motor should diffuse between two consecutive attachments to filaments is:

$$
D_{a v g}=\frac{D_{a v g 1}+D_{a v g 2}}{2}
$$

Next, we derive the jump size probability distribution. Since it is more understandable, we find the distribution of the jump process, shown by $J$, in the discrete space defined for the DTMC approximation. In what follows, we calculate the jump size on the $x$ axis. First, consider the case where the polarities of a motor and the filament do not match (the motor does not move towards the nano-relay). We divide this scenario into two parts: a motor attached to a filament other than $M_{d}$, and a motor attached to $M_{d}$. Starting with the former one, Fig. 9 shows an arbitrary scenario. $\Delta x$ is the spatial step in the DTMC approximation method. Considering Fig. 9, if the motor attaches to the region defined by $r_{1}$, when the motor finishes moving on the filament, the displacement of the motor $(\Delta d)$ will be equal to one unit. Therefore, the displacement of a motor due to its movement on a filament is equal to one unit with probability:

$$
P(\Delta d=1)=P\left(\text { motor attaches to } r_{1}\right) P\left(\theta_{1}<\theta<\theta_{2}\right) \text {, }
$$

where $\theta_{1}$ and $\theta_{2}$ are shown in Fig. 9. Filaments of different nanorelays make different angles with the $x$ axis. Different angles lead to different motor displacements on the $x$ axis caused by moving along the filament. $\theta_{1}$ and $\theta_{2}$ define the range that lead to a displacement of $j \Delta x$. Assuming a motor attaches to different positions on a filament with equal probability, we have:

$$
P\left(\text { motor attaches to } r_{1}\right)=\frac{r_{1}}{L}
$$

$r_{1}$ is the ratio of a filament located $(j-1) \Delta x$ away from the nano-relay. Swiping $\theta$ from $\theta_{1}$ to $\theta_{2}, r_{1}$ will change. However, choosing a small enough $\Delta x$, the change is small enough to be ignored. Also, since the angle between a filament and the $x$ axis is uniformly distributed, we have:

$$
P\left(\theta_{1}<\theta<\theta_{2}\right)=\frac{\theta_{2}-\theta_{1}}{2 \pi},
$$

where:

$$
\begin{aligned}
& \theta_{1}=\operatorname{acos}\left(\frac{\left(\frac{\Delta x}{2}+j \Delta x\right)}{L}\right), \\
& \theta_{2}=\operatorname{acos}\left(\frac{\left(\frac{\Delta x}{2}+(j-1) \Delta x\right)}{L}\right) .
\end{aligned}
$$

A similar approach can be used to derive the probability of one unit displacement for all possible intervals of $\theta$, and sum them to find the probability of jump size equal to one, if a motor attaches to a filament other than $M_{d}$. Similarly, the probability distribution of different jump sizes can be found. We call this probability distribution $J_{1}$.

What if a motor attaches to an $M_{d}$ ? The only difference will be in $\theta$, since for $M_{d}, \theta=0$. Having defined $\theta$, the same approach as before could be used to find jump size probability distribution if a motor attaches to an $M_{d}$. We call this probability distribution $J_{2}$.

Assuming a motor attaches to the filaments attached to a nano-relay with equal probability, the probability distribution of the jump size for the case where the polarity of a motor and the filament it is attached to are not in match is:

$$
J_{n}=\frac{M-1}{M} J_{1}+\frac{1}{M} J_{2} .
$$

Clearly, the assumption of equal probability for a motor attaching to the filaments of a nano-relay is not exact. But, as we will see in the results, this will not affect the model much.

Fig. 10(a) shows the probability distribution function of $J_{n}$ for a motor attached to a nano-relay with $10 \mu \mathrm{m}$ long filaments and $M_{d}$ of the nano-relay is directed towards the right end receiver.

We assume the jump process along $x$ and $y$ axes are independent. Considering the model is a probabilistic analysis of motors propagation, this is a reasonable assumption. Therefore, a similar method is used to find the jump distribution on the $y$ axis.

Now, if the polarity of a motor and the filament are in match, the motor starts walking towards the nano-relay. Being received, a motor is put on the filament directed towards the closest receiver $\left(M_{d}\right)$ and moves away. We divide this relocation of the motor into two parts: the relocation before it reaches the nano-relay, and the relocation after it is put on $M_{d}$. The probability distribution for the first part can be derived using a similar approach as discussed before. The second part of the relocation is deterministic. Since a motor received by a nano-relay will be put on $M_{d}$ and the receivers are the two side boundaries of the channel, the second part of the relocation will be equal to $L$ on the $x$ axis, and 0 on the $y$ axis. We call this probability distribution $J_{p}$. Fig. 10 (b) shows the probability distribution function of the jump size of a motor whose polarity is in match with the filament it is attached to, on the $x$ axis. In Fig. 10(b), the length of the filament is $10 \mu \mathrm{m}$ and the $M_{d}$ of the considered nano-relay is directed towards the right end receiver. Considering nano-relays are distributed uniformly between $\mathrm{R}+$ and $\mathrm{R}$ - topology, the overall jump size distribution is:

$$
J= \begin{cases}J_{n} & \text { with probability } \frac{1}{2} \\ J_{p} & \text { with probability } \frac{1}{2}\end{cases}
$$

Having calculated all the needed probabilities, we build the transition matrix of the molecular propagation model for 


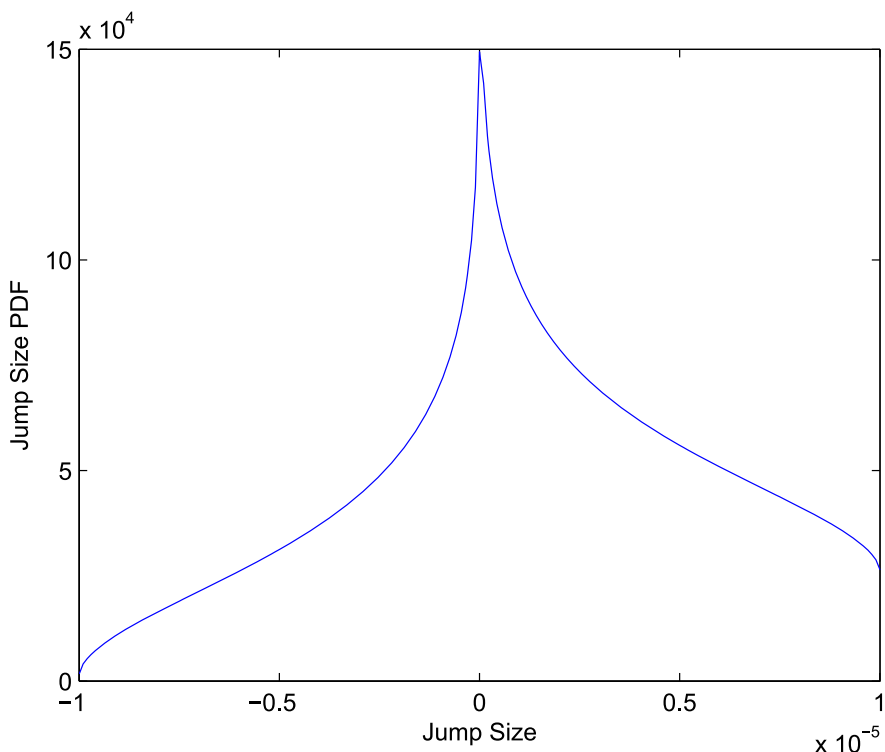

(a)

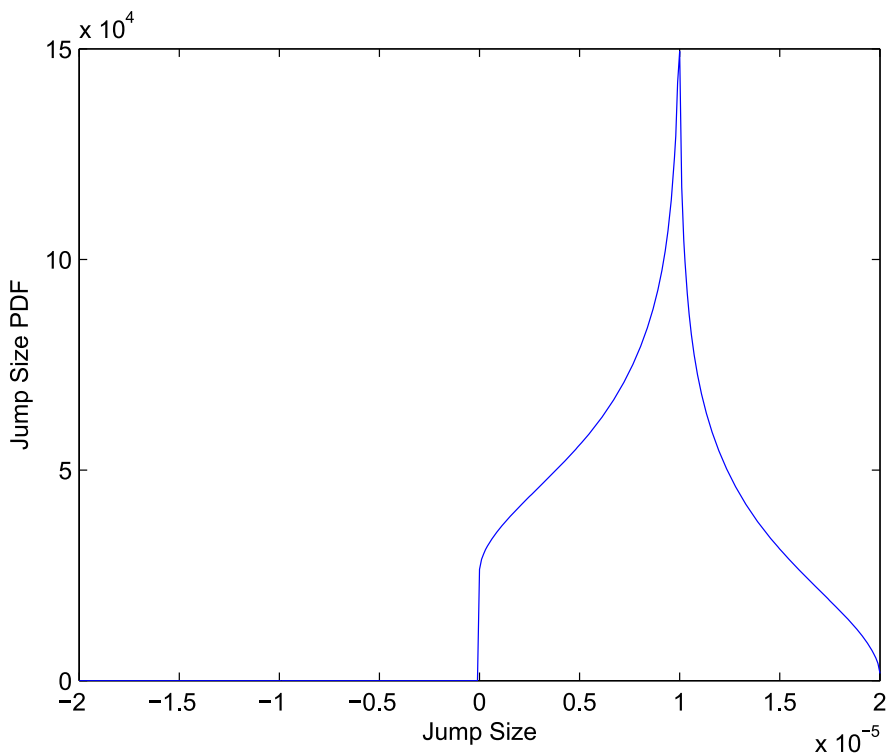

(b)

Fig. 10. Probability distribution function for jump size (length of filaments are $10 \mu \mathrm{m}$ ). (a) Motor moves towards the nano-relay. (b) Motor moves away from the nano-relay.

the molecular communication via filaments using nano-relays based on the matrix structure defined in Section III. However, one point is left. We note that the average speed assumed for a moving motor along a filament is $v=10(\mu \mathrm{m} / \mathrm{s})$. Therefore, it takes $r / v$ seconds for a motor to move $r$ meters on a filament. We should modify the proposed model to capture this issue, since in the model each jump takes one unit of time $(\Delta t)$, where based on the DTMC approximation method discussed in Section III-A, is equal to:

$$
\Delta t=\Delta x^{2} .
$$

Consider $\Delta t_{p}$ equal to the average time a motor is attached to a filament (having calculated $J$, and assuming $v$ is known, it is straightforward to find $\Delta t_{p}$ ), and choose $\Delta x_{p}$ based on (28). Defining $\Delta x_{p}$ as the spatial step, the average time a motor is attached to a filament will be equal to the time unit of the model, and the issue of a mismatch between the time unit of the model and the time interval a motor is attached to a filament is solved.

Having developed the model based on a DTMC approximation for a jump diffusion process, we discuss how the model can be used to derive a phase type distribution representing molecular delay in molecular communication via filaments using nano-relays. Consider $T_{\text {markov }}$ as the transition matrix of the Markov chain modeling molecular propagation. Clearly, the Markov chain defines the position of a motor as long as its position is between the boundaries. Assume a simple case of one-dimensional channel divided into five intervals (states). Transition matrix of the Markov chain standing for molecular propagation in the channel is:

$$
T_{\text {markov }}=\left[\begin{array}{ccccc}
1 & 0 & 0 & 0 & 0 \\
p_{2,1}^{A} & p_{0} & p_{1} & p_{2} & p_{2,5}^{A} \\
p_{3,1}^{A} & p_{-1} & p_{0} & p_{1} & p_{3,5}^{A} \\
p_{4,1}^{A} & p_{-2} & p_{-1} & p_{0} & p_{4,5}^{A} \\
0 & 0 & 0 & 0 & 1
\end{array}\right],
$$

where $p_{r}=S_{i, i+r}, r=-2,-1,0,1,2\left(S_{i, j}\right.$ defined in (10)), and $p_{i, 1}^{A}$ is the probability of absorbing to state 1 from state $i$, calculated as:

$$
p_{i, 1}^{A}=(1-\lambda \Delta t)\left(\frac{1}{2}-\frac{1}{2} q_{0}-\sum_{r=1}^{i-2} q_{r}\right)+\lambda \Delta t \sum_{r=i-1}^{J_{\text {max }}} \lambda \Delta t J_{r},
$$

$p_{i, 5}^{A}$ is the probability of absorbing to state 5 from state $i$, calculated as:

$$
p_{i, 5}^{A}=(1-\lambda \Delta t)\left(\frac{1}{2}-\frac{1}{2} q_{0}-\sum_{r=1}^{i} q_{r}\right)+\lambda \Delta t \sum_{r=i+1}^{J_{\max }} \lambda \Delta t J_{r},
$$

$q_{r}=P(X=r \Delta x)$ defined in (1), and $J_{\max }$ is the maximum jump size.

Molecular delay is the time distribution for the Markov chain to absorb to any of the absorbing states. Therefore, if we consider $T_{m p}, t^{0}$, and $\alpha_{m p}$ as:

$$
\begin{aligned}
T_{m p} & =\left[\begin{array}{ccc}
p_{0} & p_{1} & p_{2} \\
p_{-1} & p_{0} & p_{1} \\
p_{-2} & p_{-1} & p_{0}
\end{array}\right], \\
t^{0} & =\left[\begin{array}{ll}
p_{2,1}^{A} & p_{2,5}^{A} \\
p_{3,1}^{A} & p_{3,5}^{A} \\
p_{4,1}^{A} & p_{4,5}^{A}
\end{array}\right] \times[11], \\
\alpha_{m p} & =\left[\begin{array}{lll}
a_{2} & a_{3} & a_{4}
\end{array}\right],
\end{aligned}
$$

where $\alpha_{m p}$ is the distribution of the initial state (position) of a motor (we assume initial position of a motor is not at any of the absorbing states), $\left(\alpha_{m p}, T_{m p}\right)$ will be a phase type distribution representing time to absorption, or delay, of the motor. It should be noted that since PH distribution is defined as time until absorption of a Markov chain with only one absorbing state, we defined $t^{0}$ as the way shown at (33). More specifically, we considered both absorbing states as one state. This is valid because in our scenario, it is not important that the original Markov chain has absorbed to which of the absorbing states.

Considering the above explanation, the delay distribution of the motor is:

$$
\begin{aligned}
p(k) & =p\{\text { delay }=k \text { units of time }\} \\
& =\alpha_{m p}\left(T_{m p}\right)^{k-1} t^{0} .
\end{aligned}
$$


We will use this distribution to find the results in the next section.

Note that in the next Section we compare analytic and simulation results. In the simulations, the transmitter releases a motor at time zero. Brownian motion is used to find dislocation of the motor before it reaches a filament. As time goes on, it is checked whether the motor has reached a filament. If yes, instead of Brownian motion, a movement with fixed speed with the direction of the filament is assumed for the motor until it reaches the nano-relay. Then, the movement will also be with fixed speed, directly towards the closest receiver (since the nano-relay puts the motor on the filament oriented towards the closest receiver). Upon reaching the end of the filament, the motor will start diffusing again. This goes on until the motor reaches one of the receivers. It is worth mentioning that it is assumed there are enough ATP molecules in the medium and if a motor hits a filament it will attach to the filament. Also, it is assumed motors do not detach from filaments unless they reach the end of the filament.

\section{RESUlts}

In this section, we use the model to investigate the performance of the channel in terms of delay and error probability. First, we validate the model by comparing the analytical and simulation results. We find the propagation delay distribution function for two different settings of the channel based on the model and simulations, and show that the two sets of results match. Simulations are performed in MatLab. The channel in Fig. 2 with dimensions of $50 \mu \mathrm{m} \times 50 \mu \mathrm{m}$ is considered for simulations. The values assigned to parameters for the simulations are as follows. The diffusion coefficient is 1.0982 $\times 10^{-12} \mathrm{~m}^{2} / \mathrm{s}$. The average speed of motors moving on filaments is $10 \mu \mathrm{m} / \mathrm{s}$. We consider two settings for the channel and show that the model can track the changes. As the first scenario, the rate of the Poisson point process is $10 \times 10^{9} 1 / \mathrm{m}^{2}$ (average of 25 nano-relays in the channel), the number of filaments attached to each nano-relay is 4 , and the lengths of the filaments are $10 \mu \mathrm{m}$. Fig. 11(a) shows the comparison between analytical and simulation results for the scenario. As it is shown in Fig. 11(a), the analytical and simulation results are in good match. Also, we included the results for the diffusion process (in this setting, there are no nano-relays, and a released information molecule diffuses through the medium until it reaches one of the receivers). We include this setting as a reference.

In the second scenario, the rate of the Poisson point process is $6 \times 10^{9} 1 / \mathrm{m}^{2}$ (average of 15 nano-relays in the channel), the number of filaments attached to each nano-relay is 6 and the length of them is $7 \mu \mathrm{m}$. We changed all the parameters. Fig. 11(b) shows the comparison between the analytical and simulation results. The model tracks the performance of the channel well. The presented results validate the model and imply that jump diffusion process is capable of modelling the molecular propagation in molecular communication via filaments using nano-relays. In the following results, the setting of the channel is as follows, unless it is stated otherwise: the rate of the Poisson point process is $8 \times 10^{9} 1 / \mathrm{m}^{2}$ (average of 20 nano-relays in the channel), the number of filaments attached to each nano-relay is 4 , and the length of them is $10 \mu \mathrm{m}$.

Fig. 12(a)-(c) show the delay performance of the channel. Fig. 12(a) represent the delay of the channel if filaments with different length are used. If longer filaments are deployed in the channel, not only the probability that a diffusing motor attaches

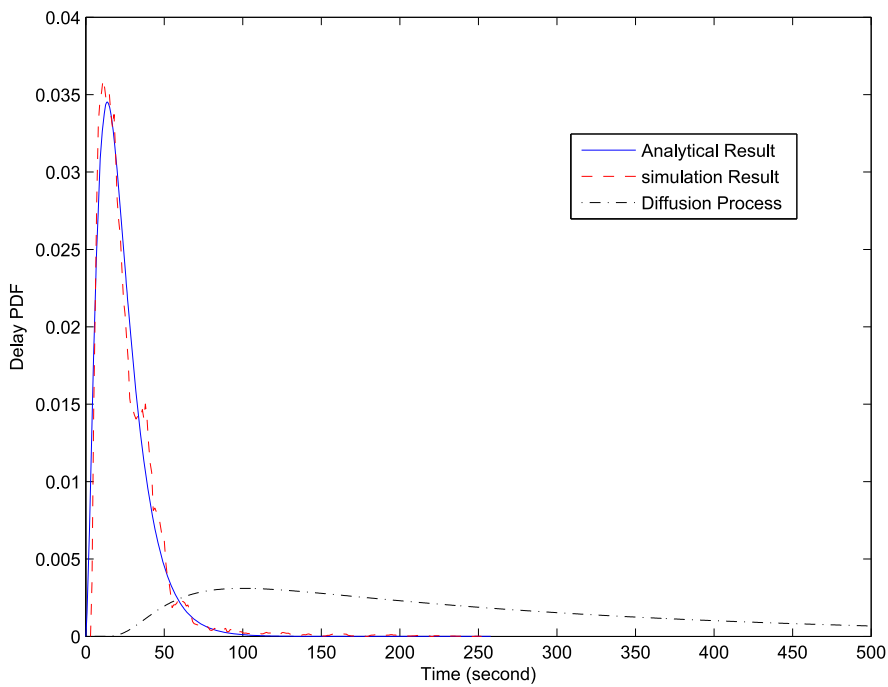

(a)

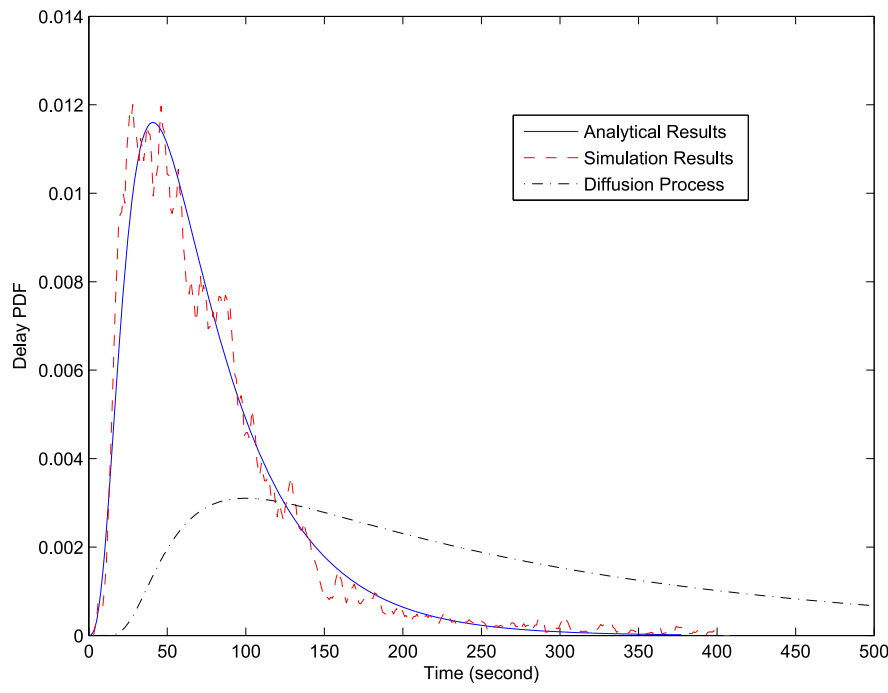

(b)

Fig. 11. Analytical results vs. simulation results. (a) Rate of Poisson point process is $10 \times 10^{9} 1 / \mathrm{m}^{2}, 4$ filaments attached to each nano-relay, with length $10 \mu \mathrm{m}$. (b) Rate of Poisson point process is $6 \times 10^{9} 1 / \mathrm{m}^{2}, 6$ filaments attached to each nano-relay, with length $7 \mu \mathrm{m}$.

to any of them will increase, but also an attached motor will be guided towards a receiver for a longer distance. Therefore, increasing the length of the filaments will decrease the delay of the channel.

Fig. 12(b) illustrates performance of the channel in terms of the delay for different number of filaments attached to each nano-relay. The probability that a motor attaches to a filament will increase if the number of filaments attached to a nano-relay increases, which will result in higher probability of guiding motors towards a receiver, and decreasing the delay.

Finally, Fig. 12(c) shows the delay for two spatial rates of the nano-relays distribution. As shown in the figure, increasing number of the nano-relays in the channel enhances the performance of the channel in terms of the delay by increasing the probability that a motor attaches to a filament.

The bit error probability is an important issue in communication. We investigate this issue in molecular communication via filaments using nano-relays. For the channel in Fig. 2, a specific modulation is considered and the average bit error probability is 


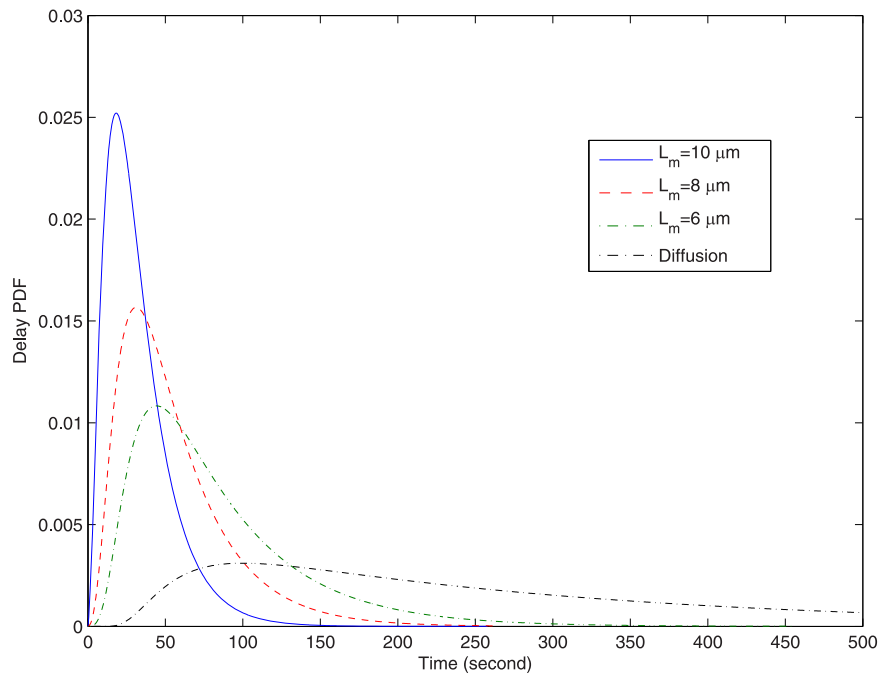

(a)

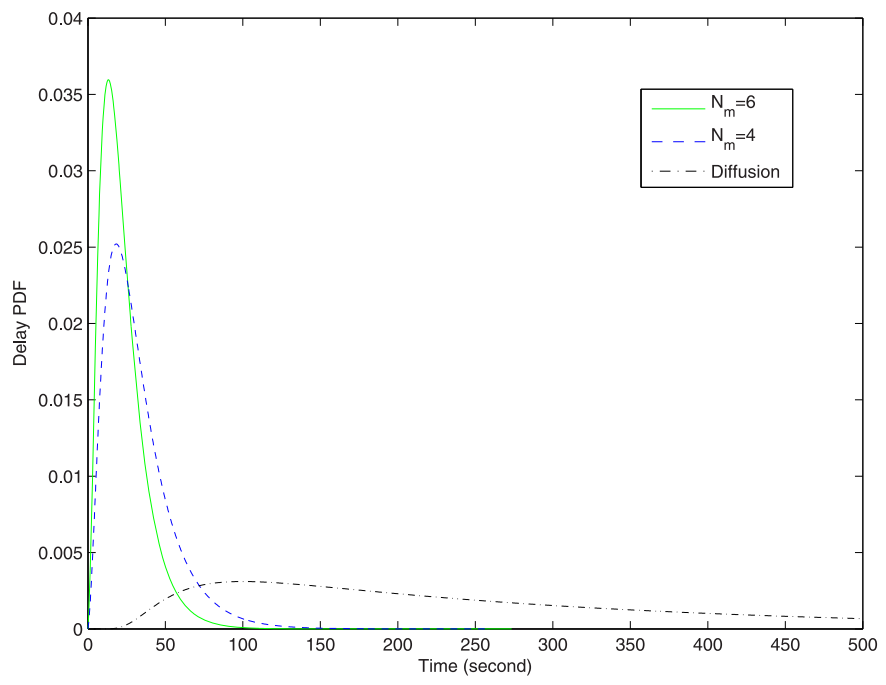

(b)

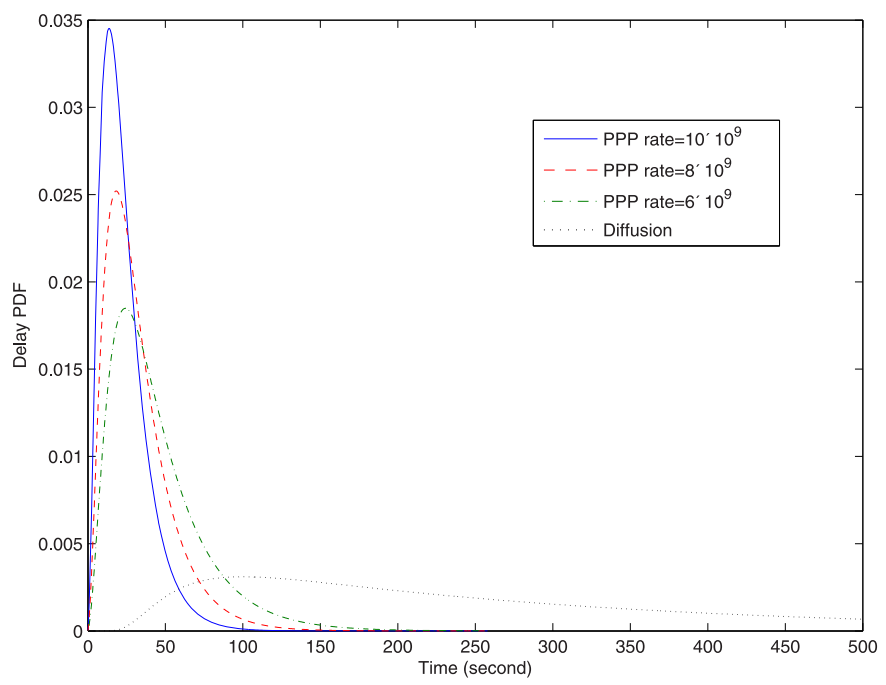

(c)

Fig. 12. Delay probability distribution. (a) Different length of filaments. (b) Different number of filaments attached to nano-relays. (c) Different rate for Poisson point process.

derived. The same approach could be used to find bit error probability of different modulation techniques. We assume the trans- mitter releases one information molecule attached to a motor as bit 1 , and does not release any molecule as bit 0 . Considering $T_{i n t e r}$ as the inter-symbol duration, we assume a released molecule is received at most $2 T_{\text {inter }}$ after its release. Of course, if it is not received in $T_{\text {inter }}$ seconds, an error may happen. In other words, we assume inter-symbol interference is caused only by the last transmitted signal. This can be justified by assuming molecular messaged dissolve in the channel after $2 T_{\text {inter }}$ seconds. For this modulation technique, the average bit error probability is:

$$
p_{\text {error }}=p_{\text {error }{ }^{\prime} 1} p(1)+p_{\text {error } \mid 0} p(0),
$$

where $p(0)$ is probability of sending bit $0, p(1)$ is probability of sending bit 1 , and:

$$
\begin{aligned}
& p_{\text {error } \mid 1}=p(0) p_{\text {int }}+p(1)\left(1-p_{\text {int }}\right) p_{\text {int }}, \\
& p_{\text {error } \mid 0}=p(1) p_{\text {int }}
\end{aligned}
$$

where $p_{\text {int }}$ is the probability that a released molecule does not reach either of the receivers in $T_{\text {inter }}$ seconds. Assuming $p(0)=$ $p(1)=1 / 2$, and finding $p_{\text {int }}$ from propagation delay distribution function, we derive the error probability. We note that since nano-relays do not decode messages and only put the arrived molecule on the filament oriented towards the closest receiver, no error is generated by nano-relays.

Fig. 13(a)-(c) show average error probability versus intersymbol duration. Fig. 13(a) illustrates the effect of the length of filaments on the average bit error probability, Fig. 13(b) shows the error probability as a function of the number of filaments attached to a nano-relay, and Fig. 13(c) illustrates error probability for different spatial rates of nano-relays. In all cases, a similar discussion as was presented for delay distribution can be done to elaborate on the reason for performance improvements.

As the last set of results, we investigate the trade off between increasing the length of filaments or the number of filaments attached to nano-relays. Assume 4 filaments with length of 6 $\mu \mathrm{m}$ are already attached to each nano-relay. We assume there are enough tubulins for each nano-relay to grow $12 \mu \mathrm{m}$ of filaments (tubulins are a kind of proteins, which are building blocks of filaments). The question is which one is better in terms of average bit error probability: growing two new filaments with length of $6 \mu \mathrm{m}$, or growing current filaments for $9 \mu \mathrm{m}$ ? Fig. 14 shows the average bit error probability for both improvements. As expected, both decisions reduce error probability. However, growing the current filaments is more beneficial for improving the average bit error probability.

\section{CONCLUSION}

In this paper, we proposed the use of nano-relays in molecular communication via filaments in free space as a technique to improve performance of this type of nanocommunication in terms of delay and bit error probability. The main goal of this paper was modeling molecular propagation in such systems. Considering the properties of the propagation, jump diffusion process was a good candidate. However, since jump diffusion processes are not investigated thoroughly, we used an approximation method based on DTMCs for jump diffusion processes and proposed the model. The model was then validated through simulations, and was used to investigate performance of the channel in terms of delay and bit error probability. It was shown that longer and more filaments will improve performance of a 


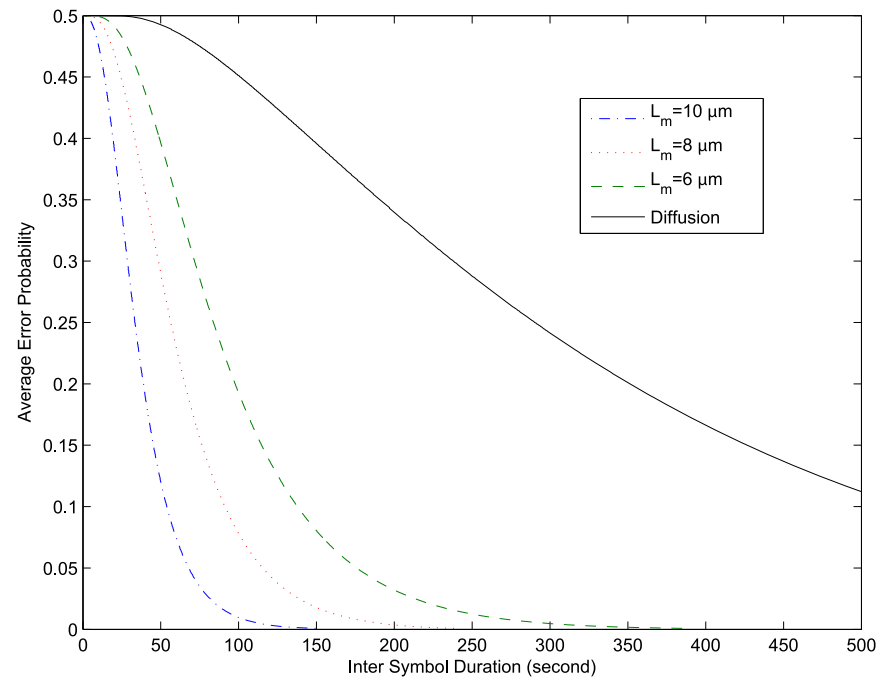

(a)

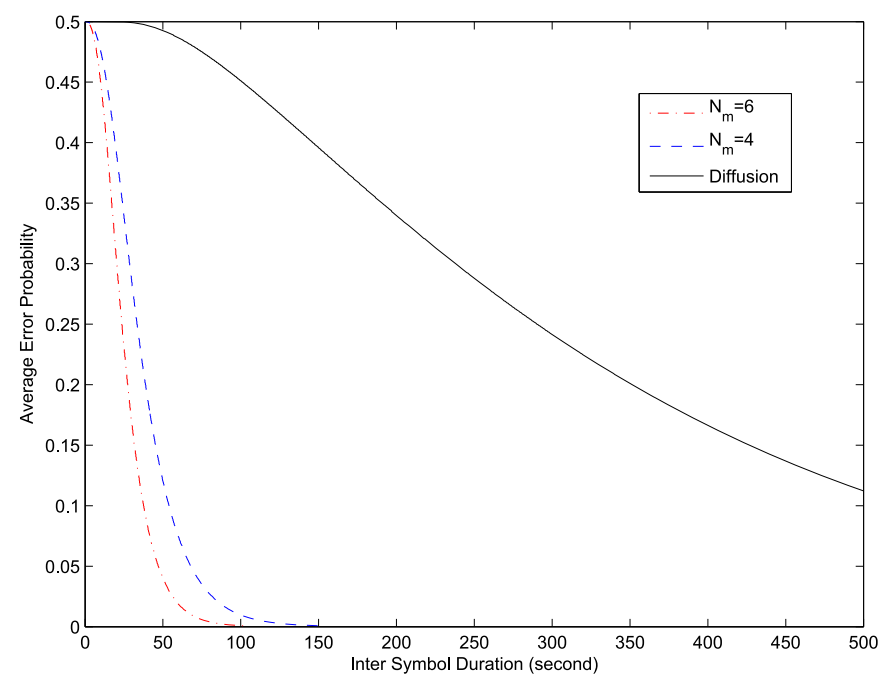

(b)

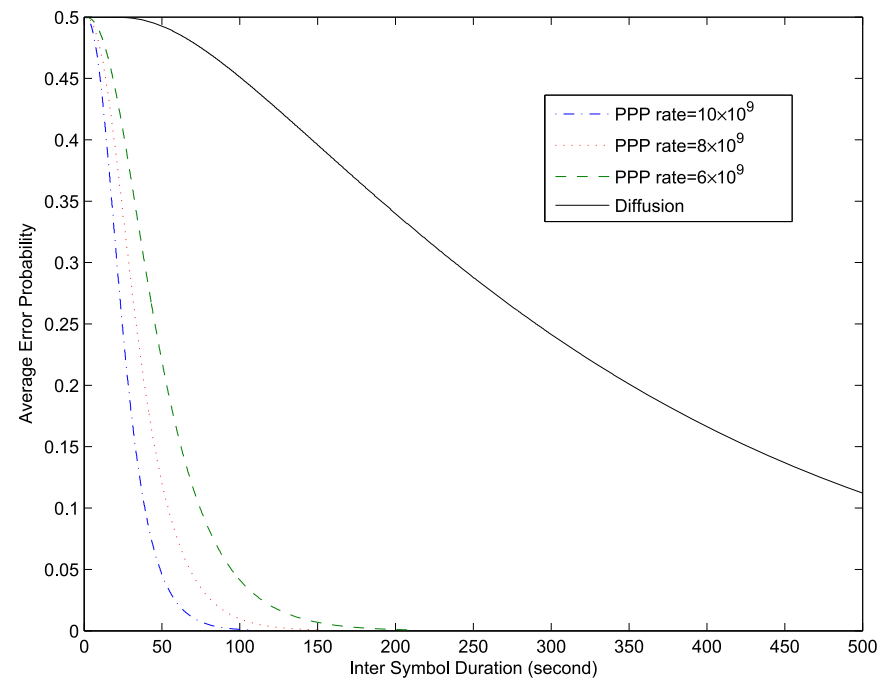

(c)

Fig. 13. Average error probability distribution. (a) Different length of filaments. (b) Different number of filaments attached to nano-relays. (c) Different rate for Poisson point process.

channel in terms of the mentioned parameters. Also, for the considered scenario using longer filaments is more efficient than

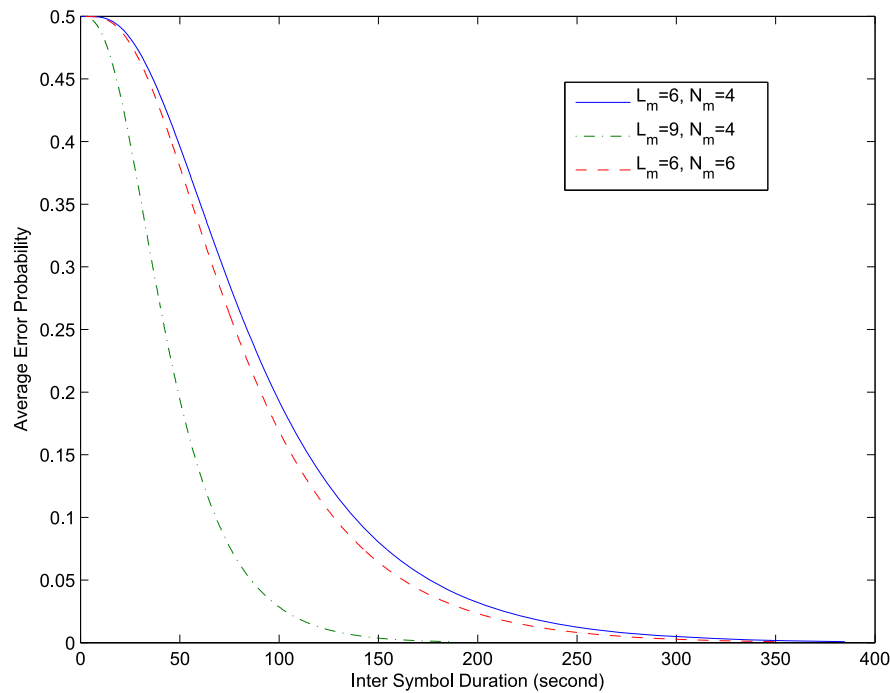

Fig. 14. Comparing the effect of the length and the number of attached filaments attached to each nano-relays in terms of average bit error probability.

adding to the number of filaments (considering the same amount of building blocks for the two scenarios). The authors are hoping this model will be useful in analyzing systems going to be implemented.

\section{REFERENCES}

[1] I. Akyildiz, F. Brunetti, and C. Blazquez, "Nano-networks: A new communication paradigm," Comput. Netw., vol. 52, no. 12, pp. 2260-2279, Aug. 2008.

[2] K. Darchini and A. S. Alfa, "Molecular communication via microtubules and physical contact in nano-networks: A survey," Nano Commun. Netw., vol. 4, no. 2, pp. 73-85, Jun. 2013.

[3] T. Nakano, M. J. Moore, F. Wei, A. V. Vasilakos, and J. Shuai, "Molecular communication and networking: Opportunities and challenges," IEEE Trans. NanoBiosci., vol. 11, no. 2, pp. 198-207, Jun. 2012.

[4] L. P. Gineé and I. F. Akyildiz, "Molecular communication options for long range nano-networks," Comput. Netw., vol. 53, no. 16, pp. 2753-2766, Nov. 2009.

[5] A. W. Eckford, N. Farsad, S. Hiyama, and Y. Moritani, "Microchannel molecular communication with nanoscale carriers: Brownian motion versus active transport," in Proc. 10th IEEE Conf. Nanotechnol., 2010, pp. 854-858.

[6] N. Farsad, A. W. Eckford, S. Hiyama, and Y. Moritani, "Information rates of active propagation in microchannel molecular communication," in Proc. Int. Conf. Bio-Inspired Models Netw, Inf., Comput. Syst., 2010, pp. 16-21.

[7] A. Farsad, N. W. Eckford, S. Hiyama, and Y. Moritani, "A simple mathematical model for information rate of active transport molecular communication," in Proc. IEEE INFOCOM Workshop, 2011, pp. $473-478$.

[8] N. Farsad, A. W. Eckford, and S. Hiyama, "A mathematical channel optimization formula for active transport molecular communication," in Proc. ICC, 2012, pp. 6137-6141.

[9] M. J. Moore, A. Enomoto, T. Suda, A. Kayasuga, and K. Oiwa, "Molecular communication: Uni-cast communication on a microtubule topology," in Proc. IEEE Int. Conf. Syst., Man., Cybern. (SMC '08), Apr. 2008, pp. 18-23.

[10] M. J. Moore, A. Enomoto, T. Nakano, A. Kayasuga, H. Kojima, H Sakakibara, K. Oiwa, and T. Suda, "Molecular communication: Simulation of microtubule topology," Natural Comput., pp. 134-144, 2009.

[11] M. J. Moore, A. Enomoto, S. Watanabe, K. Oiwa, and T. Suda, "Simulating molecular motor uni-cast information rate for molecular communication," in Proc. Annu. Conf. Inf. Sci. Syst. (CISS), Mar. 2009, pp. $859-864$.

[12] M. J. Moore, T. Suda, and K. Oiwa, "Molecular communication: Modeling noise effects on information rate," IEEE Trans. NanoBiosci., vol. 8, no. 2, pp. 169-180, Jun. 2009.

[13] D. R. Cox and H. D. Miller, The Theory of Stochastic Processes. Boca Raton, FL, USA: CRC, 2000, pp. 203-210. 
[14] B. Alberts, Molecular Biology of the Cell. New York: Garland Science, 2008.

[15] Z. Mamdouh, G. E. Kreitzer, and W. A. Muller, "Leukocyte transmigration requires kinesin-mediated microtubule-dependent membrane trafficking from the lateral border recycling compartment," J. Experimental Med., vol. 205, no. 4, pp. 951-966, 2008.

[16] K. L. Weber, A. M. Sokac, J. S. Berg, R. E. Cheney, and W. M. Bement, "A microtubule-binding Myosin required for nuclear anchoring and spindle assembly," Nature, vol. 431, no. 7006, pp. 325-329, Sep. 2004.

[17] G. Karp, Cell and Molecular Biology; Concepts and Experiments. Hoboken, NJ, USA: Wiley, 2013, pp. 330-354.

[18] A. Kamal and L. S. B. Goldstein, "Principles of cargo attachment to cytoplasmic motor proteins," Current Opinion Cell Biol., vol. 14, no. 1 , pp. 63-68, 2002.

[19] V. Varga, C. Leduc, V. Bormuth, S. Diez, and J. Howard, "Kinesin-8 motors act cooperatively to mediate length-dependent microtubule depolymerization," Cell, vol. 138, no. 6, pp. 1174-1183, 2009.

[20] J. C. Fu and T. Wu, "Linear and nonlinear boundary crossing probabilities for Brownian motion and related processes," J. Appl. Prob., vol. 47, no. 4, pp. 1058-1071, 2010.

[21] $\mathrm{T}$. $\mathrm{Wu}$, "Linear and nonlinear boundary crossing probabilities for Brownian motion and related processes," Ph.D. , Univ. Manitoba, Winnipeg, MB, Canada, 2012.

[22] S. G. Kou and H. Wang, "First passage time of a jump diffusion process," Adv. Appl. Prob., vol. 35, no. 2, pp. 504-531, 2003.

[23] N. Cai, "On first passage times of a hyper-exponential jump diffusion process," Operations Res. Lett., vol. 37, no. 2, pp. 127-134, Mar. 2009

[24] G. Klein, "Mean first-passage times of Brownian motion and related problems," Proc. Roy. Soc. London A, Math. Phys. Sci., vol. 211, no. 1106, pp. 431-443, 1952.

[25] B. D. Unluturk, D. Malak, and O. B. Akan, "Rate-delay tradeoff with network coding in molecular nano-networks," IEEE Trans. Nanotechnol., vol. 12, no. 2, pp. 120-128, Mar. 2013.

[26] A. Aijaz, A. H. Aghvami, and M. R. Nakhai, "On error performance of network coding in diffusion-based molecular nano-networks," IEEE Trans. Nanotechnol., vol. 13, no. 5, Sep. 2014.

[27] A. Maansson, M. Sundberg, R. Bunk, M. Balaz, I. A. Nicholls, P. Omling, J. O. Tegenfeldt, S. Tagerud, and L. Montelius, “Actin-based molecular motors for cargo transportation in nanotechnology potentials and challenges," IEEE Trans. Adv. Packag., vol. 28, no. 4, pp. 547-555, Nov. 2005.

[28] B. Atakan, Molecular Communications and Nanonetworks: From Nature to Practical Systems. New York: Springer, 2014, p. 145.

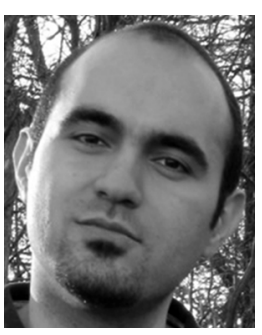

Kamaloddin Darchinimaragheh received his B.Sc. and M.Sc. degrees in electrical engineering, communication systems, from Ferdowsi University of Mashhad, Mashhad, Iran, and Sharif University of Technology, Tehran, Iran in 2008 and 2010, respectively. He is currently working towards his Ph.D. degree in the Department of Electrical and Computer Engineering at University of Manitoba, Winnipeg, Canada. His current research interests include molecular communications, stochastic modeling, Markov chains, and queuing theory.

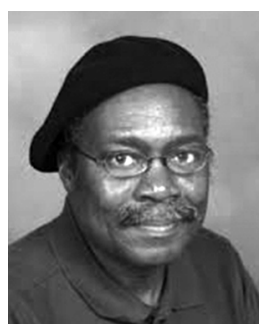

Attahiru S. Alfa is a professor of telecommunication systems at the University of Manitoba, Department of Electrical and Computer Engineering and also a SARChI Chair professor in the Department of Electrical, Electronic and Computer Engineering, University of Pretoria, Pretoria, South Africa. His research covers, but is not limited to, the following areas: queueing theory and applications, optimization, performance analysis and resource allocation in telecommunication systems, modeling of communication networks, analysis of cognitive radio networks, modeling and analysis of wireless sensor networks, developing efficient decoding algorithms for LDPC codes, channel modeling, traffic estimation for the Internet, and cross layer analysis. Dr. Alfa also works in the application of queueing theory to other areas such as transportation systems, manufacturing systems and healthcare systems. He was NSERC Chair for tele-traffic from 2004 to 2012. He has carried out applied research for Nortel Networks, Bell-Northern Research, TRLabs (now TRTech), Bell Canada, Winnipeg Regional Health Authority, Motor-coach Industries, and several other industries. Dr. Alfa teaches courses on queueing theory, telecommunication networks, optimization, and network theory. He has authored a book, Queueing Theory for Telecommunications: Discrete Time Modelling of a Single Node System, published by Springer in 2010, and has just finished another book, Applied Discrete-Time Queue, to be published in 2015 also by Springer. He co-edited two books on matrix-analytic methods published in 1996 and 1998, both based on papers of the matrix-analytic methods conferences of 1995 and 1998 , respectively. 